\begin{tabular}{|c|l|}
\hline Title & Model for cal cium-mediated reduction of structural fluctuations in epidermis \\
\hline Author(s) & Kobay ashi, Y asuaki; Kitahata, Hiroyuki; Nagay ama, Masaharu \\
\hline Citation & $\begin{array}{l}\text { Physical review E, 92(2), 022709-1-022709-7 } \\
\text { https://doi.org/L0.1103/PhysRevE.92.022709 }\end{array}$ \\
\hline Issue Date & 2015-08-13 \\
\hline Doc URL & http://hdl.handle.net/2115/59887 \\
\hline Rights & @2015A merican Physical Society \\
\hline Type & article \\
\hline File Information & PhysRevE.92.022709.pdf \\
\hline
\end{tabular}

Instructions for use 


\title{
Model for calcium-mediated reduction of structural fluctuations in epidermis
}

\author{
Yasuaki Kobayashi, ${ }^{1,2,{ }^{*}}$ Hiroyuki Kitahata, ${ }^{3}$ and Masaharu Nagayama ${ }^{1,2}$ \\ ${ }^{1}$ Research Institute for Electronic Science, Hokkaido University, Sapporo 060-0812, Japan \\ ${ }^{2}$ JST CREST, Saitama 332-0012, Japan \\ ${ }^{3}$ Department of Physics, Graduate School of Science, Chiba University, Chiba 263-8522, Japan
}

(Received 13 May 2015; published 13 August 2015)

\begin{abstract}
We propose a reaction-advection-diffusion model of epidermis consisting of two variables, the degree of differentiation and the calcium ion concentration, where calcium ions enhance differentiation. By analytically and numerically investigating this system, we show that a calcium localization layer formed beneath the stratum corneum helps reduce spatiotemporal fluctuations of the structure of the stratum corneum. In particular, spatially or temporally small-scale fluctuations in the lower structure are suppressed and do not affect the upper structure, due to acceleration of differentiation by calcium ions. Analytical expressions for the reduction rate of fluctuation amplitudes are shown.
\end{abstract}

DOI: 10.1103/PhysRevE.92.022709

\section{INTRODUCTION}

Skin is an important organ for mammals: it serves as a barrier by blocking chemical and physical attacks from outside, as well for keeping body fluid inside. The inner part of the skin is called the dermis, where blood vessels and nerve fibers exist; and the outer part is the epidermis, where epidermal cells (keratinocytes) form a layered structure. The barrier function is maintained by the outermost layers of keratinocytes, called the stratum corneum (SC), where keratinocytes have undergone terminal differentiation (cornification). An ordered structure of the $\mathrm{SC}$ is important to fully exhibit the barrier function [1].

The SC is maintained by continuous supply of epidermal cells from the basal layer, which is located along the boundary between the epidermis and the dermis. This boundary is known to be highly undulated, while the boundary between the SC and the rest of the epidermis is flat [Fig. 1(a)]. In addition, timing of stem cell division is stochastic. Therefore, in order to realize a flat boundary of the SC in spite of such spatially and temporally inhomogeneous supply of the cells, there must be a mechanism that helps coordinate the timing of cornification, which remains to be found.

Several experiments have shown that a calcium localization layer, which exists just beneath the SC, plays a vital role in epidermal homeostasis [2-4]. Motivated by these observations, we have recently proposed a mathematical model of the epidermis, which has revealed that calcium dynamics can stabilize the structure of the SC [5,6]. This model was based on particle dynamics for cell movements and reactiondiffusion process for calcium dynamics, where individual cells are assigned the degree of differentiation as an inner variable, which is affected by calcium dynamics. Because of its complexity, however, little insight has been obtained on how calcium stabilizes the structure of the SC.

In this study, we propose an analytically tractable model of the epidermis, which is based on a reaction-advectiondiffusion system. In this model, it is assumed that the differentiation process is accelerated by the presence of calcium ions and that a sustained calcium excitation is induced by

*yasuaki.kobayashi@es.hokudai.ac.jp
PACS number(s): 87.10.Ed, 82.40.Ck, 05.45.-a

(a)

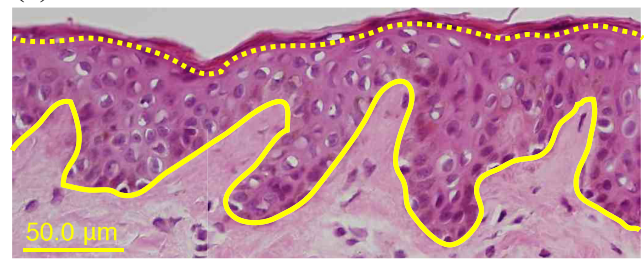

(b)

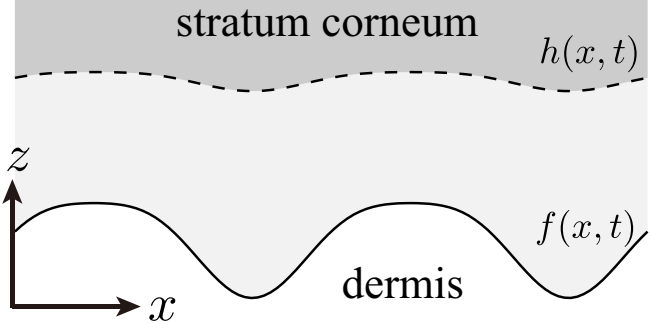

FIG. 1. (Color online) (a) Cross section of a human skin sample. The yellow (bright) solid curve is the boundary between the dermis (below) and the epidermis (above). The broken curve is the boundary between the SC (above) and the rest of the epidermis. (Image courtesy of M. Denda, Shiseido Co., Ltd., Japan.) (b) Schematic of the epidermal section, where the solid and the broken curves correspond to those in panel (a). The solid curve determines the lower interface, denoted by $z=f(x, t)$, and the broken curve determines the upper interface, denoted by $z=h(x, t)$.

cornification. We show that when the lower structure of the epidermis has fluctuations in space and time, only those with larger scales can affect the upper structure, especially the SC, and those with shorter scales are suppressed due to the effect of calcium ions. We give analytical expressions that relate the fluctuation amplitude of the upper structure to that of the lower structure.

\section{MODEL}

The following processes are known to be responsible for the formation of the stratum corneum: (a) reproduction of epidermal cells in the basal layer; (b) upward migration of 
the reproduced cells; and (c) differentiation of the cells during migration, and especially cornification as a terminal differentiation. Also, as mentioned above, (d) calcium dynamics beneath the SC could be another important factor.

As the simplest model that can describe these processes, we consider two variables, $c(x, z, t)$ and $S(x, z, t)$, representing the calcium ion distribution and the degree of differentiation, respectively, defined in a two-dimensional system. Note that individual cells are not directly considered here: In this continuous description, $c(x, z, t)$ and $S(x, z, t)$ must be understood as coarse-grained values of the cells around the point $(x, z)$. The degree of differentiation $S(x, z, t)$ specifies the state of the cells at $(x, z)$ : At $S=0$, cells are produced in the basal layer; $S$ increases in $z$ direction, which corresponds to a differentiation process during migration; and at $S=1$, cells are considered to undergo cornification. The region $S>1$ thus defines the SC.

The model is considered in the following space: $\Omega=$ $\{(x, z) \mid 0 \leqslant x \leqslant L, f(x, t) \leqslant z\}$. The boundary between the dermis and the epidermis is given by the function $f(x, t)$, which we call the lower interface. The other boundary separating the $\mathrm{SC}$ and the rest of the epidermis is denoted by $z=h(x, t)$, which we call the upper interface. By definition, $\left.S\right|_{z=f(x, t)}=0$ and $\left.S\right|_{z=h(x, t)}=1$. The lower interface is given as a boundary condition, while the upper interface is determined by $S(x, z, t)$.

The dynamics of $c(x, z, t)$ and $S(x, z, t)$ are governed by

$$
\begin{gathered}
\frac{\partial c}{\partial t}=D \nabla^{2} c-\gamma c+\sigma \delta(z-h(x, t)), \\
\frac{\partial S}{\partial t}=-U \frac{\partial S}{\partial z}+\alpha+\beta c .
\end{gathered}
$$

Upward migration is expressed as an advection term with a constant velocity $U$. A normal differentiation process is represented by $\alpha$, and when calcium exists, differentiation is accelerated by a factor $\beta$ [7]. Calcium dynamics obeys the diffusion equation with decay, which accounts for experimental observations that calcium waves in the epidermis propagate only within a finite range [5,8-10]. The source of calcium ions, which is represented by the $\delta$ function, is considered to be located along the upper interface $z=h(x, t)$, according to an experimental finding that sustained calcium excitation occurs around dead cells, which might account for the calcium localization layer beneath the SC [11]. Hence, in this model, calcium dynamics is affected by the shape of the upper boundary $h(x, t)$, which is implicitly determined by the relationship $\left.S\right|_{z=h(x, t)}=1$, while the differentiation is accelerated in the regions where the calcium concentration is high.

In the analysis below, we try to find how the upper interface $z=h(x, t)$ is determined from a given lower interface $z=$ $f(x, t)$ [see Fig. 1(b)]. It is obvious that if the dermis is flat and the migration speed is uniform in $x$, the timing of cornification is also uniform in $x$, resulting in a uniform upper interface. Hence, spatial inhomogeneity in $x$ should be caused by the different timing of cornification in $x$. It should be noted that such inhomogeneities are different from elastic deformation of the originally flat interface: Spatially uniform migration speed $U$ means that the arrangement of the cells is unchanged and hence there is no source of deformation. Therefore, the spatial structure of the SC is solely caused by the spatial dependence of $S(x, z, t)$.

The upper interface is well-defined only when $S(x, z, t)$ is a monotonically increasing function in $z$ near the region $S \sim 1$. In stationary states this is assured from Eq. (2): $\partial_{z} S=(\alpha+$ $\beta c) / U>0$. However, this is not maintained when the lower interface changes too fast in time. Throughout this paper, we only consider the case where the upper interface is always welldefined. Also we exclude cases where $f(x, t)$ is a multivalued function in $x$. Also, we consider a situation where the effect of calcium ions is well localized around the upper interface and does not affect the state around the lower interface, which is consistent with physiological situations. In other words, we consider a situation where the effect of calcium ions is negligible around the lower interface.

\section{ANALYTICAL RESULTS}

An analytical treatment is possible when the lower interface has only a small deviation from $z=0: f(z, t)=O(\epsilon)$. In this case, the upper interface $h(x, t)$ is also expected to have a small dependence on $x$ and $t$ of order $O(\epsilon)$. Let us choose a new coordinate to eliminate $h$-dependence of the $\delta$ function and simplify the analysis:

$$
\begin{gathered}
\tilde{x}=x, \\
\tilde{z}=z-h(x, t), \\
\tilde{t}=t .
\end{gathered}
$$

We also introduce new variables:

$$
\begin{aligned}
& u(\tilde{x}, \tilde{z}, \tilde{t})=u(x, z-h(x, t), t)=c(x, z, t), \\
& s(\tilde{x}, \tilde{z}, \tilde{t})=s(x, z-h(x, t), t)=S(x, z, t) .
\end{aligned}
$$

Then Eqs. (1) and (2) are rewritten as

$$
\begin{aligned}
\frac{\partial u}{\partial t}-\dot{h} \frac{\partial u}{\partial z}= & D\left\{\frac{\partial^{2}}{\partial x^{2}}-2 h^{\prime} \frac{\partial^{2}}{\partial x \partial z}-h^{\prime \prime} \frac{\partial}{\partial z}\right. \\
& \left.+\left[1+\left(h^{\prime}\right)^{2}\right] \frac{\partial^{2}}{\partial z^{2}}\right\} u-\gamma u+\sigma \delta(z), \\
\frac{\partial s}{\partial t}- & \dot{h} \frac{\partial s}{\partial z}=-U \frac{\partial s}{\partial z}+\alpha+\beta u,
\end{aligned}
$$

where $\dot{h} \equiv \partial_{t} h$ and $h^{\prime} \equiv \partial_{x} h$; and tildes of $\tilde{x}, \tilde{y}$, and $\tilde{z}$ are dropped for simplicity.

For this system we perform perturbation expansions: $u, s$, and $h$ are expanded as

$$
\begin{gathered}
u(x, z, t)=u_{0}(z)+\epsilon u_{1}(x, z, t)+O\left(\epsilon^{2}\right), \\
s(x, z, t)=s_{0}(z)+\epsilon s_{1}(x, z, t)+O\left(\epsilon^{2}\right), \\
h(x, t)=h_{0}+\epsilon h_{1}(x, t)+O\left(\epsilon^{2}\right) .
\end{gathered}
$$

Here we assume that $u$ and $s$ do not depend on $x$ and $t$ at the zeroth order.

The boundary conditions are assigned as follows. In real epidermis, calcium ions do not permeate into the SC, while the situation around the dermis is not well known. Hence, instead of imposing realistic boundary conditions, we simply assume $u(x, \pm \infty, t)=0$, and separately impose conditions 
on the model parameters so that the calcium distribution is well localized around the upper interface, as assumed above.

The boundary condition for $s$ is imposed at $z=$ $f(x, t)-h(x, t)$, which is also expanded in terms of $\epsilon$. Since $s(x, f(x, t)-h(x, t), t)=0$, by using $\partial_{z} s_{0}\left(-h_{0}\right) \sim$ $\frac{\alpha+\beta u_{0}\left(-h_{0}\right)}{U} \sim \frac{\alpha}{U}$, we have

$$
s_{0}\left(-h_{0}\right)=0, \quad s_{1}\left(x,-h_{0}, t\right)=\frac{\alpha}{U}\left\{h_{1}(x, t)-f(x, t)\right\} .
$$

Also, the cornification condition $S=1$ is now written as

$$
s_{0}(0)=1, \quad s_{1}(x, 0, t)=0 .
$$

\section{A. The zeroth order}

The zeroth order corresponds to the case $f(x, t)=0$, where the steady-state solutions $u_{0}(z)$ and $s_{0}(z)$ must satisfy

$$
\begin{gathered}
0=D \frac{\partial^{2} u_{0}}{\partial z^{2}}-\gamma u_{0}+\sigma \delta(z), \\
0=-U \frac{\partial s_{0}}{\partial z}+\alpha+\beta u_{0} .
\end{gathered}
$$

The solution $u_{0}(z)$ is obtained as

$$
u_{0}(z)=A_{0} e^{-|z| / \lambda_{\mathrm{c}}},
$$

where $A_{0} \equiv \sigma /(2 \sqrt{\gamma D})$ and $\lambda_{\mathrm{c}} \equiv \sqrt{D / \gamma} . A_{0}$ is the value of $u_{0}$ at the upper interface: $u_{0}(0)=A_{0}$. Since the value at the lower interface is written as $u_{0}\left(-h_{0}\right)=u_{0}(0) e^{-h_{0} / \lambda_{\mathrm{c}}}$, the assumption that calcium is well localized near the upper interface is expressed as

$$
e^{-h_{0} / \lambda_{\mathrm{c}}} \ll 1
$$

Substituting $u_{0}(z)$ into Eq. (16) and taking into account the boundary condition Eq. (13), $s_{0}(z)$ is also obtained as

$$
\begin{aligned}
s_{0}(z)= & \frac{\alpha\left(z+h_{0}\right)}{U}+\frac{\beta \sigma}{2 \gamma U}\left\{1-e^{-h_{0} / \lambda_{\mathrm{c}}}\right. \\
& \left.+\operatorname{sgn}(z)\left(1-e^{-|z| / \lambda_{\mathrm{c}}}\right)\right\} .
\end{aligned}
$$

The cornification condition Eq. (14) determines $h_{0}$ as

$$
1=\frac{\alpha h_{0}}{U}+\frac{\beta \sigma}{2 \gamma U}\left(1-e^{-h_{0} / \lambda_{\mathrm{c}}}\right) .
$$

Using the assumption Eq. (18), $h_{0}$ is explicitly written as

$$
\begin{gathered}
h_{0}=\frac{U}{\alpha}-C_{0} \lambda_{\mathrm{c}}, \\
C_{0} \equiv \frac{\beta A_{0}}{\alpha},
\end{gathered}
$$

where $C_{0}$ is a dimensionless parameter, which corresponds to the ratio of the speed between calcium-dependent differentiation and normal differentiation. If no calcium source exists $(\sigma=0)$, or if calcium does not affect differentiation $(\beta=0)$, the height of the upper interface is simply given by $U / \alpha$, and the height decreases due to acceleration of differentiation induced by calcium ions. The spatial profiles of $u_{0}(z)$ and $s_{0}(z)$ are shown in Fig. 2.

It should be emphasized that if we return to the original coordinates, $c_{0}(x, z, t)=u_{0}\left(z-h_{0}-h_{1}(x, t)\right)$ and $S_{0}(x, z, t)=$ $s_{0}\left(z-h_{0}-h_{1}(x, t)\right)$ contains the first order correction $h_{1}(x, t)$.
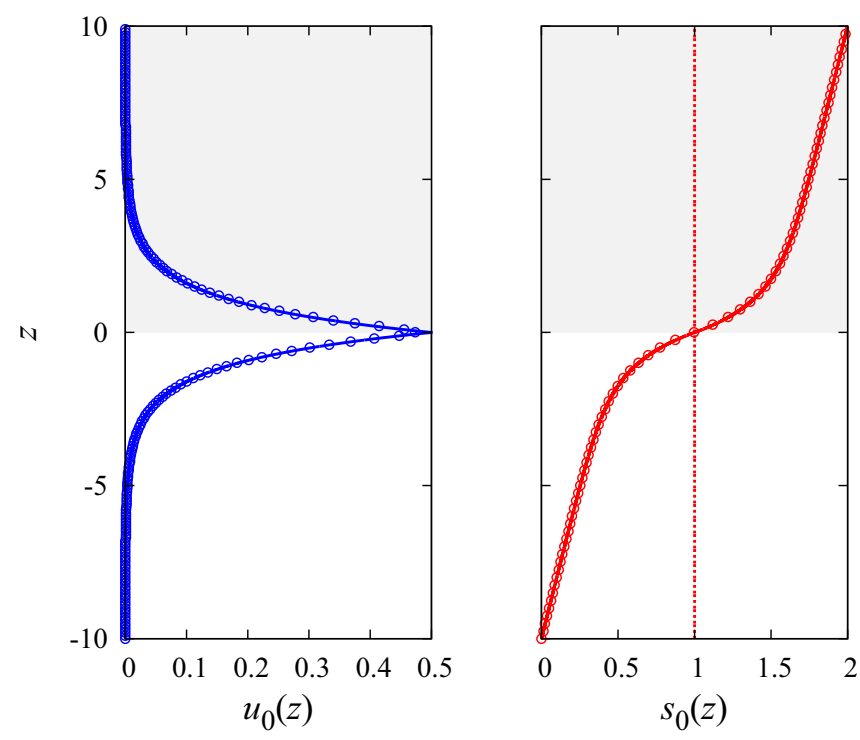

FIG. 2. (Color online) Steady-state profiles of the zeroth-order solution $u_{0}(z)$ (blue) and $s_{0}(z)$ (red), with $D=\gamma=\sigma=1, U=$ $0.01, \alpha=0.0005$, and $\beta=0.01$. Circles, numerical data; curves, analytical expressions [Eqs. (17) and (19)]. Red dotted line corresponds to $s_{0}(z)=1$. Shaded region corresponds to the SC, defined by $z$ satisfying $s_{0}(z) \geqslant 1$.

\section{B. The first order}

$$
\begin{aligned}
& u_{1}(x, z, t) \text { obeys } \\
& \left\{\partial_{t}-D\left(\partial_{x}^{2}+\partial_{z}^{2}\right)+\gamma\right\} u_{1}(x, z, t)=\left(\dot{h}_{1}-D h_{1}^{\prime \prime}\right) \partial_{z} u_{0}(z) .
\end{aligned}
$$

Since we consider only a small perturbation here, it suffices to consider a single Fourier mode of $f(x, t)$; we focus on the following case:

$$
f(x, t)=d_{q, \omega} \cos q x \sin \omega t .
$$

Then we adopt the following ansatz for the upper interface:

$$
h_{1}(x, t)=a_{q, \omega} \cos q x \sin (\omega t-\theta),
$$

and try to find the solution to Eq. (23) in the following form:

$$
u_{1}(x, z, t)=\phi_{q, \omega}(z, t) \cos q x .
$$

Substituting this into Eq. (23), $\phi_{q, \omega}(z, t)$ is obtained as

$$
\begin{aligned}
\phi_{q, \omega}(z, t)= & \frac{2 A_{0} a_{q, \omega}}{\lambda_{\mathrm{c}}}\left\{e^{-|z| / \lambda_{\mathrm{c}}} \sin \omega t\right. \\
& \left.+e^{-\kappa(q, \omega)|z|} \sin [k(q, \omega)|z|-\omega t]\right\},
\end{aligned}
$$

where

$$
\begin{gathered}
\kappa(q, \omega) \equiv \lambda_{c}{ }^{-1} \sqrt{\frac{\sqrt{\left(1+\lambda_{c}^{2} q^{2}\right)^{2}+\gamma^{-2} \omega^{2}}+1+\lambda_{c}^{2} q^{2}}{2}}, \\
k(q, \omega) \equiv \lambda_{c}-1 \sqrt{\frac{\sqrt{\left(1+\lambda_{c}^{2} q^{2}\right)^{2}+\gamma^{-2} \omega^{2}}-\left(1+\lambda_{c}^{2} q^{2}\right)}{2}} .
\end{gathered}
$$


$s_{1}(x, z, t)$ is determined from

$$
\left(\partial_{t}+U \partial_{z}\right) s_{1}(x, z, t)=\dot{h}_{1} \partial_{z} s_{0}(z)+\beta u_{1}(x, z, t) .
$$

We try to obtain $s_{1}(x, z, t)$ in the form

$$
s_{1}(x, z, t)=\left[P_{q, \omega}(z) \cos \omega t+Q_{q, \omega}(z) \sin \omega t\right] \cos q x .
$$

From Eqs. (30) and the cornification condition Eq. (14), $P_{q, \omega}(z)$ and $Q_{q, \omega}(z)$ are explicitly obtained, where the values at $z=-h_{0}$ are particularly important:

$$
\begin{aligned}
P_{q, \omega}\left(-h_{0}\right)= & \left\{\Xi_{1}(q, \omega) \sin \frac{\omega h_{0}}{U}+\Xi_{2}(q, \omega) \cos \frac{\omega h_{0}}{U}\right\} \\
& \times \frac{\alpha a_{q, \omega}}{U}, \\
Q_{q, \omega}\left(-h_{0}\right)= & \left\{\Xi_{1}(q, \omega) \cos \frac{\omega h_{0}}{U}-\Xi_{2}(q, \omega) \sin \frac{\omega h_{0}}{U}+1\right\} \\
\times & \frac{\alpha a_{q, \omega}}{U},
\end{aligned}
$$

where

$$
\begin{gathered}
\Xi_{1}(q, \omega)=-\left(1+C_{0}\right)+C_{0}\left\{\kappa(q, \omega) \chi_{1}(q, \omega)\right. \\
\left.+\left[k(q, \omega)-\frac{\lambda_{c} \omega}{U}\right] \chi_{2}(q, \omega)\right\}, \\
\Xi_{2}(q, \omega)=C_{0}\left\{\left[k(q, \omega)-\frac{\lambda_{c} \omega}{U}\right] \chi_{1}(q, \omega)-\kappa(q, \omega) \chi_{2}(q, \omega)\right\},
\end{gathered}
$$

and

$$
\begin{aligned}
& \chi_{1}(q, \omega)=\lambda_{\mathrm{c}} \frac{\frac{\lambda_{\mathrm{c}}{ }^{2} \omega^{2}}{U^{2}}+\lambda_{\mathrm{c}}{ }^{2} q^{2}+1}{\left(\frac{\lambda_{\mathrm{c}}{ }^{2} \omega^{2}}{U^{2}}+\lambda_{\mathrm{c}}{ }^{2} q^{2}+1\right)^{2}+\gamma^{-2} \omega^{2}}, \\
& \chi_{2}(q, \omega)=\lambda_{\mathrm{c}} \frac{\gamma^{-1} \omega}{\left(\frac{\lambda_{\mathrm{c}}{ }^{2} \omega^{2}}{U^{2}}+\lambda_{\mathrm{c}}{ }^{2} q^{2}+1\right)^{2}+\gamma^{-2} \omega^{2}} .
\end{aligned}
$$

Then, using the boundary condition Eq. (13), we finally obtain the relationship between the amplitudes of the lower and the upper interfaces:

$$
\frac{a_{q, \omega}}{d_{q, \omega}}=\frac{1}{\sqrt{\Xi_{1}(q, \omega)^{2}+\Xi_{2}(q, \omega)^{2}}} .
$$

This ratio depends on a length scale $\lambda_{\mathrm{c}}$, two time scales $\gamma^{-1}$ and $U^{-1} \lambda_{\mathrm{c}}$, and a dimensionless parameter $C_{0}$.

In the case of real epidermis, it is expected that the timescale of calcium dynamics is much faster than that of differentiation. Therefore, it is reasonable to assume $\gamma^{-1} \ll U^{-1} \lambda_{\mathrm{c}}$ and take $\gamma^{-1} \omega \rightarrow 0$. In this limit, the ratio of the amplitude is characterized by only three parameters $C_{0}, \lambda_{\mathrm{c}}$, and $U^{-1} \lambda_{\mathrm{c}}$, and Eq. (38) is simplified as

$$
\frac{a_{q, \omega}}{d_{q, \omega}}=\frac{1}{\sqrt{\left(1+C_{0}\right)^{2}+\frac{C_{0}^{2}-2 C_{0}\left(1+C_{0}\right) \sqrt{1+\lambda_{\mathrm{c}}^{2} q^{2}}}{1+\lambda_{\mathrm{c}}^{2} q^{2}+U^{-2} \lambda_{\mathrm{c}}^{2} \omega^{2}}}} .
$$

In particular, the following limiting behavior is obtained:

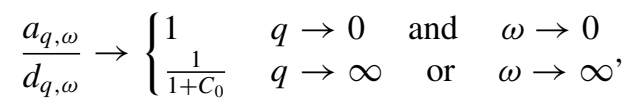

where the limit of $\omega$ is taken while keeping $\gamma^{-1} \omega \ll U^{-1} \lambda_{c} \omega$. Thus, we find that when the lower interface changes in space and time, high-wave-number or high-frequency modes are suppressed by the factor $1 /\left(1+C_{0}\right)$.

The characteristic scales of the length and the time that separate the two limiting behaviors are determined by solving the conditions $a_{q, 0} / d_{q, 0}=1 / 2$ and $a_{0, \omega} / d_{0, \omega}=1 / 2$ with respect to $q$ and $\omega$, respectively. Denoting the solutions by $q^{*}$ and $\omega^{*}$, we find

$$
\begin{gathered}
q^{*}=\frac{\sqrt{2 C_{0}-1} \frac{1}{C_{0}-1},}{\lambda_{\mathrm{c}}}, \\
\omega^{*}=\frac{\sqrt{3}}{\sqrt{C_{0}^{2}+2 C_{0}-3}} \frac{U}{\lambda_{\mathrm{c}}},
\end{gathered}
$$

suggesting that the critical spatial scale is determined by the diffusion length of calcium ions $\lambda_{c}$, and the critical time scale is determined by $U^{-1} \lambda_{\mathrm{c}}$, the time needed to move the distance $\lambda_{\mathrm{c}}$ with the velocity $U$. Note, however, that these scales are modified by the factors that are decreasing functions of $C_{0}$. Thus, the dimensionless parameter $C_{0}$ affects both the amplitude ratio and the critical scales. As mentioned before, $C_{0}$ corresponds to the effect of calcium ions on differentiation. Therefore, this result means that as the effect of calcium ions becomes stronger, undulations with larger and larger spatial and time scales are suppressed, and the suppression rate itself becomes larger.

There are several constraints to the parameter values: in addition to the calcium localization condition [Eq. (18)] and the above-mentioned separation of the timescale $\gamma^{-1} \ll U^{-1} \lambda_{\mathrm{c}}$, the thickness of the epidermis must be finite: $h_{0}>0$. Since $C_{0}$ also affects the entire thickness of the epidermis [Eq. (21)], too large $C_{0}$ collapses the epidermis.

Since we can choose units of length, time, and calcium concentration arbitrarily, only three parameters are independent in this system, and we can find such parameter values that satisfy all these constraints: For example, Eq. (39) is plotted in Fig. 3 with $D=\gamma=\sigma=1, U=0.01, \alpha=0.0005$, and $\beta=0.01$, which yields $\lambda_{\mathrm{c}}=1$ and $C_{0}=10$. The yellow (bright) solid curve is given by $a_{q, \omega} / d_{q, \omega}=1 / 2$, where the $q$ and $\omega$ intercepts are given by $q^{*}$ and $\omega^{*}$, respectively. In the present choice of the parameters, $q^{*}=0.48 \lambda_{\mathrm{c}}{ }^{-1}$ and $\omega^{*}=0.16 U \lambda_{\mathrm{c}}{ }^{-1}$.

\section{Numerical simulation}

We numerically solve Eqs. (1) and (2) for two special cases, $\omega=0$ or $q=0$, with different amplitudes of $f(x, t)$. The parameters are the same as in the plot for Fig. 3. We choose the time step as 0.0001 , grid size in $x$ and $z$ as 0.2 and 0.005 , respectively, and the explicit Euler method is adopted. The $\delta$ function is approximated by $\delta(z)=\frac{1}{\Delta \sqrt{\pi}} e^{-z^{2} / \Delta^{2}}$. Here, $\Delta=0.01$ is found to provide good accuracy, which has been confirmed by comparing numerical results of $u_{0}(z)$ and $s_{0}(z)$ to analytical ones (see Fig. 2). 


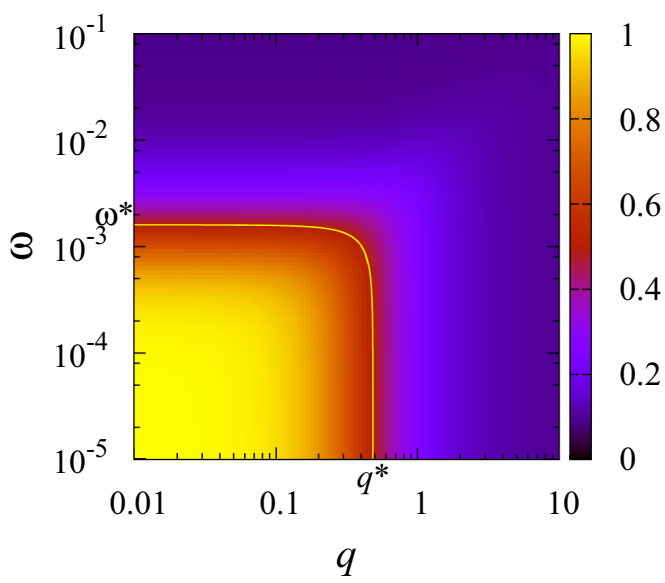

FIG. 3. (Color online) Ratio of the amplitudes $a_{q, \omega} / d_{q, \omega}$ against $q$ and $\omega$, obtained from Eq. (39), with $D=\gamma=\sigma=1, U=0.01, \alpha=$ 0.0005 , and $\beta=0.01$. The yellow (bright) solid curve corresponds to $a_{q, \omega} / d_{q, \omega}=1 / 2$, whose $q$ and $\omega$ intercepts are given by Eqs. (41) and (42), respectively.

Figures 4 and 5 show the numerical results in the case of $\omega=0$ and $q=0$, respectively. In both cases, when the amplitude is small, the numerical results are in excellent agreement with the theoretical curves. When the amplitude becomes larger, the ratio deviates from the analytical results, but the overall tendency, i.e., suppression of the amplitude in high $q$ and $\omega$ regions, is preserved. Large deviations in low $q$ and $\omega$ regions come from the fact that the amplitude of the upper interface is so large that the calcium localization condition [Eq. (18)] is no longer valid. Also in Fig. 5, data points cannot be extended to high-frequency regions: when $\omega d_{0, \omega} / U>1, s(x, z, t)$ is no longer a monotonically increasing function at near $s=0$, and if it is not monotonous near $s=1$, the upper interface $z=h(x, t)$ is ill-defined.

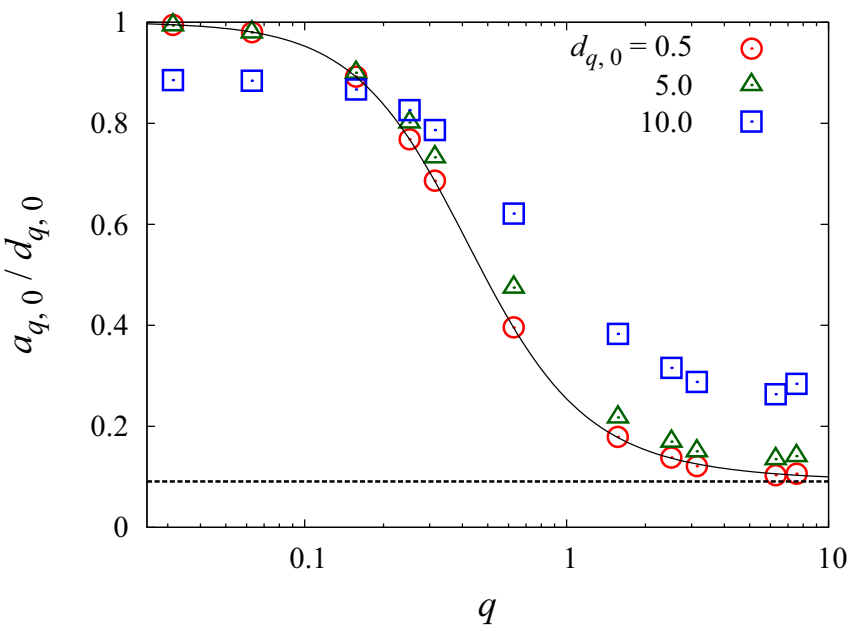

FIG. 4. (Color online) Ratio of the amplitudes $a_{q, 0} / d_{q, 0}$ in the case of time-independent lower interface $f(x)=d_{q, 0} \cos q x$, as a function of $q$, with $D=\gamma=\sigma=1, U=0.01, \alpha=0.0005$, and $\beta=$ 0.01 . Numerical data (circles, squares, and triangles) are obtained for three different values of $d_{q, 0}$; the solid curve is obtained from Eq. (39) with $\omega=0$; the dotted line is $\frac{1}{1+C_{0}}=0.091$. Simulations were performed until steady states were reached.

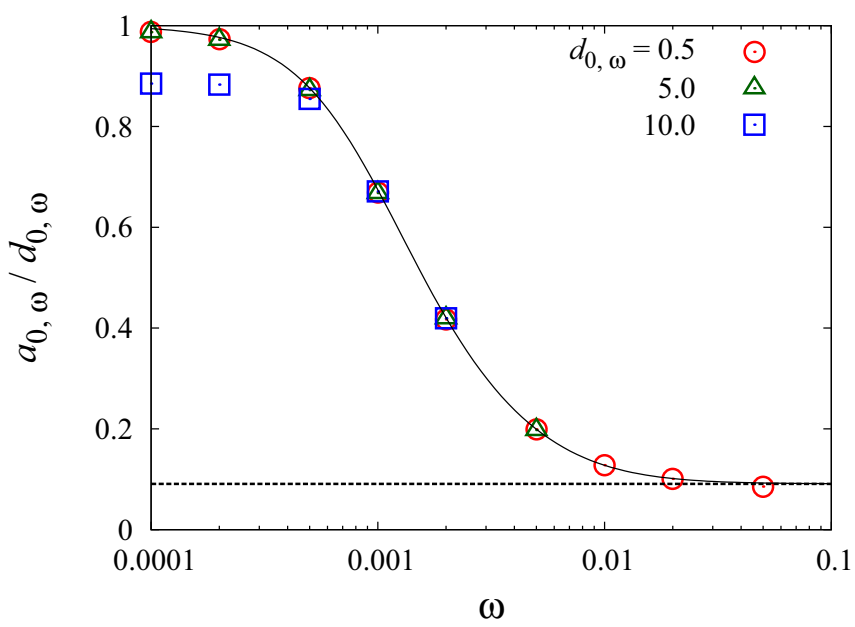

FIG. 5. (Color online) Ratio of the amplitudes $a_{0, \omega} / d_{0, \omega}$ in the case of spatially uniform lower interface $f(t)=d_{0, \omega} \sin \omega t$, as a function of $\omega$, with $D=\gamma=\sigma=1, U=0.01, \alpha=0.0005$, and $\beta=$ 0.01 . Numerical data (circles, squares, and triangles) are obtained for three different values of $d_{0, \omega}$; the solid curve is obtained from Eq. (39) with $q=0$. The dotted line is $\frac{1}{1+C_{0}}=0.091$. Simulations were performed until periodic solutions were obtained.

\section{DISCUSSION AND SUMMARY}

Let us check that the length and the time scales derived from our parameter choice are consistent with experimental observations. Without loss of generality, we can set $D=\gamma=$ $\sigma=1$, as we did in numerical simulations. This is equivalent to saying that we set the units of the length and the time as $\lambda_{c}=$ $\sqrt{D / \gamma}=1$ and $\gamma^{-1}=1$, respectively. Then there remain three model parameters: $U, \alpha$, and $\beta$; or equivalently, the model can be characterized by three dimensionless parameters: $h_{0} / \lambda_{c},\left(h_{0} / U\right) / \gamma^{-1}$, and $C_{0}$ [Eqs. (21) and (22)], the first two of which can be estimated from observations.

The first parameter $h_{0} / \lambda_{c}$ is the thickness of the epidermis measured by the diffusion length of calcium ions. From an experimental fact that the calcium localization layer beneath the SC is one cell diameter [2-4], one can estimate that the diffusion length $\lambda_{c}$ is comparable to the cell size $d$. On the other hand, although the thickness of the epidermis varies from place to place, a typical size is of the order $10 d$, as is found in Fig. 1(a), which must be equated with $h_{0}$. Therefore, $h_{0} / \lambda_{c}=$ 10 calculated from our parameter set is a reasonable choice.

The second parameter $\left(h_{0} / U\right) / \gamma^{-1}$ is the cell migration time compared to the time scale of epidermal calcium dynamics. It is known to be approximately 12 days for an epidermal cell to migrate from the basal layer to the SC [12], while a typical timescale of epidermal calcium dynamics is of the order of minutes. Our parameter choice yields $\left(h_{0} / U\right) / \gamma^{-1}=0.001$, which corresponds to the timescale of calcium dynamics estimated as $\sim 17 \mathrm{~min}$, which is acceptable as a crude order estimation.

On the other hand, the remaining parameter $C_{0}$, which is the ratio between the speed of calcium-assisted differentiation and that of normal differentiation, cannot be estimated directly from observations. Therefore, we have treated this as a free parameter. As mentioned above, $C_{0}$ changes the characteristic length scale $l^{*} \equiv 2 \pi / q^{*}$ and the time scale $t^{*} \equiv 2 \pi / \omega^{*}$ 
[Eqs. (41) and (42)], as well as determining the limiting value of the amplitude ratio as $1 /\left(1+C_{0}\right)$. For $C_{0}=10$, as chosen above, we obtain $l^{*} / \lambda_{c}=12.9, t^{*} / \gamma^{-1}=3.92 \times 10^{3}$, and $1 /\left(1+C_{0}\right)=0.09$. This means that spatial fluctuations comparable to or smaller than the thickness of the epidermis are reduced with the reduction rate up to $9 \%$. This sounds reasonable, judging from Fig. 1(a) and other experimental observations. Also, temporal fluctuations up to three orders of magnitude longer than calcium dynamics, and in particular those coming from calcium dynamics themselves, are well suppressed by the same factor.

We have proposed a mechanism for the formation of the spatially and temporally uniform SC by the acceleration of differentiation due to the calcium ion distribution beneath the SC. In particular, due to this mechanism, small spatial and temporal scales are filtered out and the SC has only large-scale fluctuations, which is in accord with experimental observations. Although we have only considered fluctuations coming from the lower interface, in reality, there might be fluctuations of differentiation during the migration process, or those of calcium distribution. However, such fluctuations can be short scales in space and time, and are expected to be suppressed by the same mechanism.

Our simple model does not take into account various aspects of epidermis: In real epidermis, epidermal cells are tightly bound. Also, rigidity of the SC and the change of the cell shape along the differentiation process are neglected. However, although these features surely contribute to the structural stability of the epidermis by providing resilience from deformations of the originally flat structure, they cannot be principal factors for producing the flat SC structure from the beginning: the default structure of the SC could be already spatially inhomogeneous, if cornification occurs at different altitudes. Our model suggests a way of controlling the timing of cornification by calcium-dependent differentiation, thereby producing a flat structure of the SC.

We have assumed for simplicity that the upward migration $U$ is constant in space and time. The spatial homogeneity of migration is partly justified due to the fact that cell adhesion prevents the rearrangement of the neighboring cells except in the vicinity of the basal layer, where adhesion is weak and cells can be rearranged so that they are supplied uniformly to the upper layers. However, when the supply of the cells from the basal layer largely changes in space and time, migration is no longer treated as constant. Since such an inhomogeneous cell supply is considered to occur in skin disorder such as psoriasis, extension of our model to space-time dependence of $U$ will be of practical importance.

\section{ACKNOWLEDGMENT}

We are grateful to Professor K. Nakamura for valuable comments.

\section{APPENDIX: STABILITY ANALYSIS FOR THE ZEROTH-ORDER SOLUTION}

Here we show that the position of the upper interface at the zeroth order, $h=h_{0}$, is stable against perturbation to the zeroth-order solutions $u=u_{0}$ and $s=s_{0}$.
Suppose that small perturbations are applied to the steady state $u=u_{0}(z)$ and $s=s_{0}(z)$ at $t=0$. It suffices to consider a mode containing $e^{i k x}$ in $x$ direction. As a result of the perturbations, $u$ and $s$ deviate from the steady state:

$$
\begin{gathered}
u(x, z, t)=u_{0}(z)+e^{i k x} \xi(z, t), \\
s(x, z, t)=s_{0}(z)+e^{i k x} \eta(z, t) .
\end{gathered}
$$

By the perturbations, the upper interface is also shifted from the steady state: $h(x, t)=h_{0}+e^{i k x} H(t)$, where $H(t)$ is determined through the relation $\eta\left(-h_{0}, t\right)=\alpha H(t) / U$. The deviations $\xi(z, t)$ and $\eta(z, t)$ obey

$$
\begin{gathered}
\left\{\partial_{t}+D k^{2}-D \partial_{z}^{2}+\gamma\right\} \xi(z, t)=\left(\dot{H}+D k^{2} H\right) \partial_{z} u_{0}(z) \\
\left(\partial_{t}+U \partial_{z}\right) \eta(z, t)-\beta \xi(z, t)=\dot{H} \partial_{z} s_{0}(z) .
\end{gathered}
$$

If $H$ is treated as a given function, Eqs. (A3) and (A4) are inhomogeneous linear equations, and the solutions $\xi(z, t)$ and $\eta(z, t)$ have terms that explicitly contain the initial conditions $\xi(z, 0)$ and $\eta(z, 0)$, and other terms that contain $H$. It is obvious that the $\eta(z, 0)$ term is advected away for $t \gtrsim h_{0} / U$, and the $\xi(z, 0)$ term decays for $t \gtrsim \gamma^{-1}$. Therefore, after a transient period, the effect of initial perturbations remains only through $H$, and it suffices to confirm that these $H$ terms, which are obtained as the particular solutions to Eqs. (A3) and (A4), decay in time. Let us try to find them in the form $\xi(z, t)=$ $e^{\lambda t} \tilde{\xi}(z)$ and $\eta(z, t)=e^{\lambda t} \tilde{\eta}(z)$, with $H(t)=e^{\lambda t} \tilde{H}$. Substituting these into Eqs. (A3) and (A4), we obtain

$$
\begin{gathered}
\lambda_{\mathrm{c}} \frac{\tilde{\xi}(z)}{\tilde{H}}=A_{0}\left(e^{\frac{z}{\lambda_{\mathrm{c}}}}-e^{\sqrt{1+\gamma^{-1} \lambda+\lambda_{\mathrm{c}}{ }^{2} k^{2}} \frac{z}{\lambda_{\mathrm{c}}}}\right), \\
\frac{U}{\alpha} \frac{\tilde{\eta}(z)}{\tilde{H}}=1+C_{0} e^{\frac{z}{\lambda_{\mathrm{c}}}}-C_{0} \frac{e^{\sqrt{1+\gamma^{-1} \lambda+\lambda_{\mathrm{c}}{ }^{2} k^{2}} \frac{z}{\lambda_{\mathrm{c}}}}}{\frac{\lambda_{\mathrm{c}}}{U} \lambda+\sqrt{1+\gamma^{-1} \lambda+\lambda_{\mathrm{c}}{ }^{2} k^{2}}} \\
-e^{-\frac{\lambda z}{U}}\left(1+C_{0}-\frac{C_{0}}{\frac{\lambda_{\mathrm{c}}}{U} \lambda+\sqrt{1+\gamma^{-1} \lambda+\lambda_{\mathrm{c}}{ }^{2} k^{2}}}\right),
\end{gathered}
$$

for $z \leqslant 0$. Using the condition $\tilde{\eta}\left(-h_{0}\right)=\alpha \tilde{H} / U$ and the assumption of calcium localization $e^{-h_{0} / \lambda_{\mathrm{c}}} \sim 0$, we obtain the eigenvalue equation

$$
0=1+C_{0}-C_{0} \frac{1-e^{-\left(\frac{\lambda}{U}+\sqrt{1+\gamma^{-1} \lambda+\lambda_{\mathrm{c}}^{2} k^{2}}\right) \frac{h_{0}}{\lambda_{\mathrm{c}}}}}{\frac{\lambda_{\mathrm{c}}}{U} \lambda+\sqrt{1+\gamma^{-1} \lambda+\lambda_{\mathrm{c}}{ }^{2} k^{2}}}
$$

For $\lambda \geqslant 0$, the exponential term is smaller than $e^{-h_{0} / \lambda_{\mathrm{c}}}$ and can be neglected. Then the right-hand side becomes greater than $C_{0}>0$. Therefore, no solution exists for $\lambda \geqslant 0$. Therefore, the deviation of the upper interface $h_{1}(t)$ decays in time, and we can conclude that the zeroth-order steady state is stable. 
[1] P. M. Elias and K. R. Feingold, in Skin Barrier, edited by P. M. Elias and K. R. Feingold (Taylor \& Fransis, New York, 2006), p. 1.

[2] A. Sakuntabhai, V. Ruiz-Perez, S. Carter, N. Jacobsen, S. Burge, S. Monk, M. Smith, C. S. Munro, M. O’Donovan, N. Craddock, R. Kucherlapati, J. L. Rees, M. Owen, G. M. Lathrop, A. P. Monaco, T. Strachan, and A. Hovananian, Nature Genet. 21, 271 (1999).

[3] T. Mauro, G. Bench, E. Sidderas-Haddad, K. Feingold, P. Elias, and C. Cullander, J. Invest. Dermatol. 111, 1198 (1998).

[4] M. Denda, J. Hosoi, and Y. Asida, Biochem. Biophys. Res. Commun. 272, 134 (2000).

[5] Y. Kobayashi, Y. Sanno, A. Sakai, Y. Sawabu, M. Tsutsumi, M. Goto, H. Kitahata, S. Nakata, J. Kumamoto,
M. Denda, and M. Nagayama, PLOS ONE 9, e92650 (2014).

[6] Y. Kobayashi, Y. Sawabu, H. Kitahata, M. Denda, and M. Nagayama (unpublished).

[7] E. Fuchs, J. Cell Biol. 111, 2807 (1990).

[8] M. Tsutsumi, K. Inoue, S. Denda, K. Ikeyama, M. Goto, and M. Denda, Cell Tissue Res. 338, 99 (2009).

[9] N. J. Warren, M. H. Tawhai, and E. J. Crampin, Exp. Physiol. 95, 232 (2009)

[10] J. R. Edwards and W. G. Gibson, J. Theor. Biol. 263, 45 (2010).

[11] M. Tsutsumi, M. Goto, and M. Denda, Exp. Dermatol. 22, 367 (2013).

[12] H. Iizuka, J. Dermatol. Sci. 8, 215 (1994). 